\title{
EL TRATAMIENTO PERIODÍSTICO DEL SUICIDIO EN DIARIOS DIGITALES DE ARGENTINA, ESPAÑA Y PERÚ
}

\section{THE JOURNALISTIC TREATMENT OF SUICIDE IN DIGITAL NEWSPAPERS OF ARGENTINA, SPAIN AND PERU}

Silvia Granja-González y María Dolores Meneses-Fernández

| silvia.granja.08@ull.edu.es | dmeneses@ull.edu.es |

Universidad de La Laguna

Resumen: En este trabajo analizamos el tratamiento periodístico de cuatro casos de suicidio de personajes famosos internacionales en siete diarios digitales editados en tres países hispanohablantes: Argentina, España y Perú. Los dos sudamericanos regulan a través de sus códigos deontológicos el tratamiento del suicidio. En España, ningún código deontológico lo hace. Los casos seleccionados de Chester Bennington, Robin Williams, Tony Scott y Alexander McQueen garantizaban el seguimiento de la noticia y el hallazgo de un número de noticias representativo. Nuestro objetivo fue identificar el grado de adecuación de los medios a las normas de autorregulación y a las recomendaciones de profesionales de la salud. Aplicamos el análisis de contenidos periodísticos, de códigos deontológicos y de documentos internacionales elaborados por expertos en salud. Los resultados revelan la disfunción en el tratamiento informativo y las normas deontológicas respecto a las directivas internacionales. Palabras clave: suicidio; tratamiento periodístico; deontología periodística; Sudamérica; España. 


\begin{abstract}
This work shows an analysis of the journalistic treatment of four cases of suicide of international celebrities in digital newspapers of three Spanish-speaking countries: Argentina, Spain and Peru. Two of them, Argentina and Peru, regulate the treatment of suicide through their deontological codes. In Spain, none of the deontological codes applied at the national level do so. The chosen cases have been those of Chester Bennington, Robin Williams, Tony Scott and Alexander McQueen. Our goal was to identify the degree of adequacy of the media to self-regulation norms and to the recommendations of health professionals. We did an analysis of journalistic content, deontological codes and international guidelines prepared by health experts. The results reveal the dysfunction in the informative treatment and the deontological norms regarding the international directives. Keywords: suicide; journalistic treatment; journalistic ethics; South America; Spain.
\end{abstract}

\title{
1. Introducción y antecedentes
}

Los suicidios se pueden prevenir $^{1}$, afirma la primera línea del prefacio del informe Preventing suicide. A global imperative de la Organización Mundial de la Salud (OMS, 2014). Según los datos de dicho informe, los suicidios suponen más de 800.000 muertes al año en el mundo, lo que supone que cada 40 segundos se suicida una persona. Estas cifras lo sitúan como una de las principales causas de muerte violenta en el mundo. Al tratar su prevención, la OMS incide en el papel determinante que desempeñan los medios de comunicación.

En España se suicidaron 3.679 personas en 2017 (datos del Instituto Nacional de Estadística, www.ine.es). En 2015, hubo 3.602 suicidios, lo que supone una tasa de 7,76 por 100.000 habitantes que aumentó a 8 en 2016. Desde 2008 estas muertes superan las causadas por accidentes de tráfico. Los informativos españoles dan cuenta cada lunes del número de fallecidos en accidentes de tráfico, pero la cobertura de las muertes por suicidio es casi nula.

Según la OMS $(2017,2018)$, en 2015 se suicidaron 6.166 personas en Argentina, una tasa de 14,29 por 100.000 habitantes, reducida a 9,2 en 2016. En Perú se registraron 1.381 suicidios en 2015, una tasa de 5,86 por 100.000 habitantes que disminuyó a 4,9 en 2016.

El suicidio ha sido percibido históricamente de forma negativa en la cultura occidental. Platón lo consideró un hecho al margen de cualquier norma establecida, tanto de carácter religioso, legal o político (Clemente y González, 1996). Para Aristóteles era un «acto no propio del valiente» y pensaba que iba contra la sociedad (De la Vega, 1996). Fueron San Agustín y Santo Tomás de Aquino quienes establecieron su vínculo con el cristianismo, al considerar

[01] Traducción del original en inglés. 
que si Dios otorgaba vida al hombre, solo Él tenía la potestad de truncarla (Clemente y González, 1996).

Dado que analizamos el tratamiento mediático del suicidio en Argentina, España y Perú, cabe recordar que son países de tradición católica, en los que el tabú y hermetismo en torno al acto suicida persiste aún. La tradición cultural configuró esta percepción negativa y el prejuicio a través de prácticas como quemar los cadáveres de quienes se suicidaban o arrojarlos a los ríos (Barbero, 1966). En los territorios de la Corona de Castilla, Alfonso XI decretó, en el siglo XIV, que los suicidas perdían el derecho a legar sus bienes a herederos, confiscándolos la Corona; continuó aplicándose durante el reinado de Enrique III, en el siglo XV (Barbero, 1966).

Tras la publicación en 1774 de la novela de Goethe Las penas del joven Werther, caló la idea de que hablar de suicidio podría producir un efecto de imitación. Phillips (1974) refiere que, tras leerse la novela en Europa, muchas personas imitaron la forma de suicidarse del personaje.

Pirkis y Blood (2001) explican que Phillips (1974) concluyó, a partir de sus investigaciones de 1947 y 1968, que el número de suicidios aumentaba tras la publicación en la portada de los periódicos de casos de suicidios. Consideraba el efecto contundente e inmediato y restringido a las áreas en las que el suceso era publicado.

Wasserman (1984) revisó los datos de Phillips e investigó la publicación de casos de suicidios en las portadas de The New York Times. Consideró que no había un efecto significativo, pero sí encontró un aumento considerable en los meses en los que se difundía el suicidio de un personaje famoso. Wasserman determinó que los suicidios de celebridades causaban un aumento de los suicidios mensuales porque sus muertes son más publicitadas.

En 1990, Stack (en Pirkis y Blood, 2001) comprobó que la difusión de casos de suicidios estaba directamente relacionada con el aumento de su tasa. Por otro lado, se constató que las indicaciones aplicadas en Austria para el tratamiento periodístico del suicidio redujeron su número (Pirkis y Blood, 2001).

Del suicidio de personajes famosos, Niederkrotenthaler et al. (2012) afirman que la identificación y admiración son factores clave a la hora de provocar un efecto imitador. Verificaron, a través de un meta-análisis, que la tasa de suicidios aumentaba tras la publicación de informaciones sensacionalistas sobre la muerte por esa causa de famosos. Y añadieron que informar de manera responsable de casos en los que se superaron momentos de crisis, sin recurrir a comportamientos suicidas, se relaciona directamente con la reducción de su tasa. Muestra así el doble efecto que causan los medios de comunicación. 
Desde el enfoque psiquiátrico, estudios sobre casos asiáticos mediatizados muestran que informar de manera indiscreta y excesiva del suicidio de celebridades arrastra por imitación a personas y sociedades propensas al suicidio (Cheng et al., 2007; Fu y Yip, 2009; Ju Ji et al., 2014; Park et al., 2016; Young et al., 2017).

Estos antecedentes explican el rechazo a cubrir casos de suicidio en los medios informativos porque, como señalan Beam, John y Yaqub (2017) respecto al periodismo estadounidense, muchos medios y periodistas dicen no informar sobre suicidios, salvo cuando informan de ellos. Es una paradoja extendida en las tradiciones periodísticas de Europa, América y Asia.

Las cifras convierten el suicidio en un problema de salud pública. El rechazo a hablar de suicidio en los medios y la confusión que genera en los periodistas justifican este estudio, orientado a conocer el comportamiento de los medios, y la observancia que hacen de los referentes deontológicos y de las recomendaciones elaboradas por instituciones sanitarias.

\section{Marco teórico}

La ética y deontología periodísticas, y las recomendaciones externas a la profesión periodística delimitan nuestro marco de referencia.

La ética se ocupa de cuál es el propósito de los profesionales de la información y de cómo deben actuar. En el tratamiento mediático del suicidio intervienen aspectos de la ética y deontología periodísticas que deben ser considerados en la cobertura periodística del suicidio. Olmo (2015) mantiene que los posibles límites al informar de estos casos vienen dados por la protección de la infancia, la salud pública, la intimidad, el honor, la integridad física y moral.

El derecho a la libertad de expresión e información adquiere especial relevancia para los profesionales de los medios de comunicación (Camps, 1995). Para esta autora, dos factores limitan esa libertad: no causar perjuicios a otras personas y actuar con buena voluntad.

López (2002) explica que el interés público es aquel que se corresponde con el de la mayor parte de individuos de un país, y profundiza en los casos en los que puede resultar oportuno informar sobre el dolor humano:

Al ser humano le interesan las informaciones sobre el sufrimiento ajeno en cuanto es consciente de que puede sufrirlo y afectarle particular e individualmente en algún momento de su vida. Existen casos concretos en los que el interés informativo radica, no en el dolor en sí mismo, sino en la persona concreta que padece esta aflicción, dada la relevancia pública que ostente y dado que sus actuaciones públicas en el desempeño de su 
profesión, en consecuencia, de la situación dolorosa que atraviese, pueden afectar al bien común.

Procede que los periodistas se planteen si existe un conflicto entre la trayectoria pública de las celebridades y su intimidad. En este sentido, López Mañero (1997) expone que la intimidad de los personajes públicos es más limitada que la de las personas privadas, quienes tienen mayor control sobre ella. Aun así, considera que la revelación de la intimidad debe siempre ser voluntaria. Además, subraya que no existe distinción entre el dolor y el sufrimiento que experimentan celebridades y personas anónimas. Urzúa (2003) también se ocupa de esta idea y señala que todos, personajes públicos o privados, son iguales ante la muerte y tienen el mismo derecho a mantener su intimidad. Se deduce de lo anterior que el respeto informativo es extensible a las personas fallecidas, sean anónimas o famosas.

La deontología concreta la ética periodística para regular las actuaciones profesionales, ya que ordenan los principios que sirven de guía. «Se refiere a lo que es vinculante para todos, para un colectivo profesional» (López, 2016: 19). Se traduce en textos de autorregulación que buscan extender su cumplimiento por el bien general que defienden. Un ejemplo son los códigos deontológicos, instrumentos de autorregulación por excelencia. Recogen normas obligatorias y comunes para periodistas y comunicadores (Rodríguez, 1995). Su finalidad es definir un estándar moral de la profesión, un êthos profesional (Bonete, 1995: 21).

En el ámbito hispanohablante solo hemos encontrado dos códigos nacionales que incluyen consideraciones explícitas del tratamiento del suicidio. Uno es el Código de Ética del Foro de Periodismo Argentino (Código de ética de FOPEA, 2006), que establece que «debe evitarse la publicación de suicidios, a menos que se trate de casos de ostensible valor informativo». Aunque evitan informaciones innecesarias, pueden coartar la «alfabetización en salud mental» a la que se refiere Jorm (en Ministerio de Sanidad, Política Social e Igualdad, 2011: 27). Este código subraya en su epígrafe de valores esenciales que «el buen gusto es un valor periodístico, por lo que la curiosidad escatológica, la estridencia innecesaria y la morbosidad son actitudes a evitar».

El otro código que alude al suicidio es el de la Federación de Periodistas del Perú (Vignolo, 2001). En su artículo noveno insta a evitar el sensacionalismo y establece que la forma de conseguirlo es

Ofrecer sin escándalo ni sensacionalismo la información necesaria sobre hechos trágicos, desagradables o que puedan lastimar los normales 
sentimientos humanos, ser muy cauto con la información relacionada con suicidios, violaciones y hechos negativos en los que estén involucrados, como víctimas o actores la mujer, los niños y adolescentes.

En España, el código deontológico de la Federación de Asociaciones de Periodistas de España (FAPE, 2017) no incluye recomendaciones explícitas para el tratamiento del suicidio. No obstante, es factible aplicar el epígrafe 4 (apartados a y c) de los Principios Generales que establece que «solo la defensa del interés público justifica las intromisiones o indagaciones sobre la vida privada de una persona sin su previo consentimiento»; y el alusivo al tratamiento de asuntos que comporten «elementos de dolor o aflicción en las personas afectadas, el periodista evitará la intromisión gratuita y las especulaciones innecesarias sobre sus sentimientos y circunstancias». Lo ejemplificamos con la queja que motivó la Resolución 2016/125 de la Comisión de Arbitraje, Quejas y Deontología del Periodismo (Comisión creada por iniciativa de la FAPE).

En cuanto a las recomendaciones externas a la profesión periodística, la OMS inició en 1999 el proyecto multidisciplinar SUPRE de prevención de los suicidios que condujo a la publicación de un manual de referencia. En él se incluyen recomendaciones para los medios de comunicación orientadas al tratamiento informativo adecuado de estos casos (OMS, 2000).

La OMS destaca la influencia de los medios de comunicación, de ahí que el manual conceda importancia al tratamiento mediático de los casos de suicidio. Clasifican las recomendaciones en dos grupos «Cómo informar del suicidio en general» y «Cómo informar sobre un suicidio específico». Además, insta a publicar información útil para que las personas en riesgo de quitarse la vida busquen apoyo y ayuda.

En la misma línea, publicó en 2008 y 2017 las actualizaciones de estas recomendaciones, de las que solo la versión de 2000 está traducida al español. Acosta et al. (2017) revisaron las tres versiones y concluyeron que este hecho dificulta el acceso de los periodistas de habla hispana a estos documentos.

Así mismo, este organismo remarca la necesidad de extremar la precaución al informar del suicidio de una celebridad. Los casos de famosos tienen un impacto indudable en el público, pues la vida de estas personas forma parte del imaginario colectivo. Esta relevancia pública incita a conocer su final.

El seguimiento de las recomendaciones de la OMS en medios de habla hispana ha sido estudiada en varias investigaciones (Herrera et al., 2014; Muñoz y Sánchez, 2014; Olmo y García, 2014; Müller, 2011; Pubiano et al., 2007). Los resultados revelan su escasa repercusión en el trabajo periodís- 
tico, ya sea por desconocimiento o por dejación. Estudios recientes indican un incremento en la aplicación de estas recomendaciones (Garrido, Serrano y Catalán, 2018; Lois, Rodríguez y Túñez, 2018), aunque solo de algunas de ellas y sin seguir una pauta homogénea.

Otro documento que trata la salud mental de la población y la prevención del suicidio es el de la Comisión de las Comunidades Europeas (2005). En él instan a los Estados miembro de la Unión Europea a adoptar medidas preventivas, refiriéndose de forma específica a los periodistas encargados de informar de un intento de suicidio.

En España, el Ministerio de Sanidad, Política Social e Igualdad redactó en 2011 una estrategia de salud mental que comprende desde 2009 a 2013. La atención prestada a los medios de comunicación es escasa. No obstante, los considera fundamentales para lo que Jorn et al. (2006: 3) denominan alfabetización en salud mental, un proceso por el que se forma a la población sobre aspectos básicos de las enfermedades mentales. Dicho Ministerio considera que los medios son un vehículo esencial para formar a la sociedad. Este convencimiento le llevó a elaborar una Guía Práctica en la que alude al papel de los medios de comunicación en la prevención del suicidio (Ministerio de Sanidad, Política Social e Igualdad, 2012). El Ministerio de Salud del Gobierno de Argentina (2016) también cuenta con una guía de recomendaciones basada en gran parte en las recomendaciones de la OMS.

\section{Objetivos e hipótesis}

Motiva nuestro estudio averiguar si los medios informativos del ámbito hispanohablante tratan el suicidio de personas famosas acorde con las recomendaciones de instituciones sanitarias y profesionales de la salud. Responde también al interés de saber si los códigos deontológicos orientan a los periodistas en prácticas coherentes con las recomendaciones de esas instituciones sanitarias y profesionales médicos; y si los libros de estilo que limitan la cobertura del suicidio logran mejorar las informaciones sobre estos casos. Cabría combinar nuestro enfoque ético-deontológico y profesional con normas jurídicas, pero dado que la deontología periodística debe respetar la legislación vigente resultaría redundante.

Las hipótesis del estudio son las siguientes:

$\rightarrow$ H1. Atendiendo a las pautas deontológicas y profesionales disponibles, los medios de comunicación realizan un tratamiento informativo irresponsable de los casos de suicidio de personas famosas. 
$\rightarrow$ H2. La autorregulación periodística sobre la cobertura de suicidios es tan superficial que no genera diferencias sustanciales en la calidad periodística de países con y sin autorregulación.

$\rightarrow$ H3. Los medios de comunicación con libro de estilo que limita el tratamiento del suicidio emiten menos y mejores informaciones que los demás.

\section{Material y metodología}

Hemos analizado 4 casos de suicidio de famosos publicados en periódicos digitales de difusión nacional de Argentina, España y Perú. Esta selección deriva del rastreo preliminar realizado (tabla 1), durante el que observamos qué países de habla española cuentan con normas deontológicas elaboradas por asociaciones nacionales de periodistas, destacando Argentina y Perú al contar con códigos éticos que hacen referencia al suicidio.

Tabla 1. Códigos deontológicos revisados durante el rastreo preliminar.

\begin{tabular}{|c|c|c|c|c|c|}
\hline País & Código deontológico & Organización & $\begin{array}{l}\text { Referencia } \\
\text { al suicidio }\end{array}$ & Año & Ámbito \\
\hline Argentina & Código de ética de FOPEA (2006) & \begin{tabular}{|l|} 
Foro de Periodismo \\
Argentino (FOPEA)
\end{tabular} & Sí & 2006 & Nacional \\
\hline Argentina & $\begin{array}{l}\text { Código de ética de la prensa argentina } \\
\text { (Díaz del Campo-Lozano y Chaparro- } \\
\text { Domínguez, 2017; Accountable } \\
\text { Journalism, s.f.a) }\end{array}$ & $\begin{array}{l}\text { Asociación de Entidades } \\
\text { Periodísticas Argentinas }\end{array}$ & No & 2012 & Nacional \\
\hline Bolivia & $\begin{array}{l}\text { Código de Ética de la Asociación } \\
\text { Nacional de Periodistas de Bolivia } \\
(2007)\end{array}$ & $\begin{array}{l}\text { Asociación Nacional de } \\
\text { Periodismo de Bolivia (ANP) }\end{array}$ & No & 2007 & Nacional \\
\hline Chile & $\begin{array}{l}\text { Código de Ética de Periodistas de } \\
\text { Chile (2015; Díaz del Campo-Lozano } \\
\text { y Chaparro-Domínguez, 2017) }\end{array}$ & $\begin{array}{l}\text { Colegio de Periodistas de } \\
\text { Chile }\end{array}$ & No & 2015 & Nacional \\
\hline Colombia & $\begin{array}{l}\text { Código de Ética del Círculo de } \\
\text { Periodistas de Bogotá (2006; Díaz } \\
\text { del Campo-Lozano y Chaparro- } \\
\text { Domínguez, 2017) }\end{array}$ & $\begin{array}{l}\text { Círculo de Periodistas de } \\
\text { Bogotá }\end{array}$ & No & 2006 & Regional \\
\hline Costa Rica & $\begin{array}{l}\text { Código de ética de las y los } \\
\text { profesionales de la comunicación } \\
\text { (2011; Díaz del Campo-Lozano y } \\
\text { Chaparro-Domínguez, 2017) }\end{array}$ & $\begin{array}{l}\text { Colegio de Periodistas de } \\
\text { Costa Rica }\end{array}$ & No & 2011 & Nacional \\
\hline Cuba & $\begin{array}{l}\text { Código de Ética del Periodista (2013; } \\
\text { Díaz del Campo-Lozano y Chaparro- } \\
\text { Domínguez, 2017) }\end{array}$ & $\begin{array}{l}\text { Unión de Periodistas de } \\
\text { Cuba }\end{array}$ & No & 2013 & Nacional \\
\hline Ecuador & $\begin{array}{l}\text { Código de Ética del Periodista } \\
\text { (Federación Nacional de Periodistas } \\
\text { del Ecuador, 1980; Díaz del Campo- } \\
\text { Lozano y Chaparro-Domínguez, 2017) }\end{array}$ & $\begin{array}{l}\text { Federación Nacional de } \\
\text { Periodistas del Ecuador } \\
\text { (FENAPE) }\end{array}$ & No & 1980 & Nacional \\
\hline
\end{tabular}


El tratamiento periodístico del suicidio en diarios... | Granja-González y Meneses-Fernández | 65

\begin{tabular}{|c|c|c|c|c|c|}
\hline País & Código deontológico & Organización & $\begin{array}{l}\text { Referencia } \\
\text { al suicidio }\end{array}$ & Año & Ámbito \\
\hline El Salvador & $\begin{array}{l}\text { Código de ética para los periodistas } \\
\text { y profesionales de la comunicación } \\
\text { en El Salvador (APES, 2012; Díaz } \\
\text { del Campo-Lozano y Chaparro- } \\
\text { Domínguez, 2017) }\end{array}$ & $\begin{array}{l}\text { Asociación de Periodistas } \\
\text { de El Salvador (APES) }\end{array}$ & No & 2012 & Nacional \\
\hline España & Código Deontológico (FAPE, 2017) & $\begin{array}{l}\text { Federación de Asociaciones } \\
\text { de Periodistas de España } \\
\text { (FAPE) }\end{array}$ & No & 2017 & Nacional \\
\hline Guatemala & $\begin{array}{l}\text { Código de Ética de la Asociación } \\
\text { de Periodistas de Guatemala (Díaz } \\
\text { del Campo-Lozano y Chaparro- } \\
\text { Domínguez, 2017; Accountable } \\
\text { Journalism, s.f.b) }\end{array}$ & $\begin{array}{l}\text { Asociación de Periodistas } \\
\text { de Guatemala }\end{array}$ & No & 2000 & Nacional \\
\hline Guatemala & $\begin{array}{l}\text { Código de ética del Periodista y } \\
\text { Comunicador de Guatemala (2015- } \\
\text { 2016) }\end{array}$ & $\begin{array}{l}\text { Cámara Guatemalteca de } \\
\text { Periodismo }\end{array}$ & No & $\begin{array}{l}2015- \\
2016\end{array}$ & Nacional \\
\hline Honduras & $\begin{array}{l}\text { Código de Ética (Colegio de } \\
\text { Periodistas de Honduras, 1979; } \\
\text { Díaz del Campo-Lozano y Chaparro- } \\
\text { Domínguez, 2017) }\end{array}$ & $\begin{array}{l}\text { Colegio de Periodistas de } \\
\text { Honduras }\end{array}$ & No & 1979 & Nacional \\
\hline México & $\begin{array}{l}\text { Código de Ética (Díaz del Campo- } \\
\text { Lozano y Chaparro-Domínguez, 2017; } \\
\text { Accountable Journalism, s.f.c) }\end{array}$ & - & No & 1996 & Nacional \\
\hline Nicaragua & - & - & - & - & - \\
\hline Panamá & $\begin{array}{l}\text { Código de Ética Periodística (2007; } \\
\text { Díaz del Campo-Lozano y Chaparro- } \\
\text { Domínguez, 2017) }\end{array}$ & $\begin{array}{l}\text { Fórum de Periodistas por } \\
\text { las libertades de expresión e } \\
\text { información }\end{array}$ & No & 2007 & Nacional \\
\hline Paraguay & $\begin{array}{l}\text { Código de Ética del Sindicato de } \\
\text { Periodistas del Paraguay (1999; } \\
\text { Díaz del Campo-Lozano y Chaparro- } \\
\text { Domínguez, 2017) }\end{array}$ & $\begin{array}{l}\text { Sindicato de Periodistas del } \\
\text { Paraguay (SPP) }\end{array}$ & No & 1999 & Nacional \\
\hline Perú & $\begin{array}{l}\text { Código de Ética (Díaz del Campo- } \\
\text { Lozano y Chaparro-Domínguez, 2017; } \\
\text { Accountable Journalism, s.fd) }\end{array}$ & $\begin{array}{l}\text { Asociación Nacional de } \\
\text { Periodistas }\end{array}$ & No & 1988 & Nacional \\
\hline Perú & $\begin{array}{l}\text { Código de Ética Periodística (Vignolo, } \\
\text { 2001) }\end{array}$ & $\begin{array}{l}\text { Federación de Periodistas } \\
\text { del Perú, Alfredo Vignolo } \\
\text { Maldonado }\end{array}$ & Sí & 2001 & Nacional \\
\hline \begin{tabular}{l|} 
República \\
Dominicana
\end{tabular} & $\begin{array}{l}\text { Código de Ética (1994; Díaz y } \\
\text { Chaparro-Domínguez, 2017) }\end{array}$ & $\begin{array}{l}\text { Colegio Dominicano de } \\
\text { Periodistas (CDP) }\end{array}$ & No & 1994 & Nacional \\
\hline Uruguay & $\begin{array}{l}\text { Código de Ética Periodística (2013; } \\
\text { Díaz y Chaparro-Domínguez, 2017) }\end{array}$ & $\begin{array}{l}\text { Asociación de la Prensa } \\
\text { Uruguaya (APU) }\end{array}$ & No & 2013 & Nacional \\
\hline Venezuela & $\begin{array}{l}\text { Código de Ética del Periodista } \\
\text { Venezolano (2013; Díaz y Chaparro- } \\
\text { Domínguez, 2017) }\end{array}$ & $\begin{array}{l}\text { Colegio Nacional de } \\
\text { Periodistas (CNP) }\end{array}$ & No & 2013 & Nacional \\
\hline
\end{tabular}

Fuente: elaboración propia. 
Los casos de suicidio estudiados fueron los de cuatro creadores reconocidos a nivel internacional: Alexander McQueen, diseñador británico que se suicidó el 11 de febrero de 2010; Tony Scott, director de cine que puso fin a su vida el 19 de agosto de 2012; Robin Williams, actor estadounidense que se quitó la vida el 11 de agosto de 2014; y Chester Bennington, vocalista del grupo estadounidense Linkin Park que acabó con su vida el 20 de julio de 2017. En esos años, 2010-2017, se dieron otros suicidios de celebridades (el cantante Chris Cornell, el diseñador de moda L'Wren Scott o el chef Bernard Loiseau), pero por necesidad de acotar la investigación, la centramos en cuatro casos cuyos resultados consideramos proyectables a los otros.

Los diarios digitales en los que analizamos los cuatro casos de suicidio fueron los españoles $A B C$ y El Mundo, los diarios generalistas más consultados tras El País (Statista, 2017), diario digital español que también incluimos en el estudio porque su libro de estilo es considerado una referencia profesional al ser seguido por diarios hispanohablantes de todo el mundo (Libro de Etilo El País, 2014: 10; Rubiano, Quintero y Bonilla, 2007).

$A B C$ es el principal periódico conservador en España, con una línea editorial católica y monárquica. El Mundo ha mantenido desde su fundación y hasta 2014 la línea editorial liberal de Diario 16, de donde procedía su equipo directivo (Herrero, Artero y Sánchez-Taberno, 2009). El País se autodefine como «un periódico independiente, nacional, de información general, [...]» (Libro de Estilo El País, 1996: 11).

De Argentina analizamos Clarín y La Nación, los diarios digitales más visitados mensualmente en este país (ComScore, 2017). Clarín es el diario argentino más leído, tanto en formato impreso como digital (Zunino, 2015) y su línea editorial se identifica ideológicamente con la centro-derecha argentina. La Nación es el segundo diario argentino más leído en el país; abarca el mismo espectro ideológico que Clarín, su principal competidor.

Y de Perú analizamos El Comercio y La República, los diarios nacionales más visitados en la web (IAB Perú, 2018). El Comercio es uno de los diarios peruanos históricos, y el de mayor renombre y trayectoria de Perú (García, 2011). Se sitúa en el centro-derecha del espectro político (Bello, 2014). La República se sitúa en la centro-izquierda del espectro político, comprometido con la defensa de los derechos humanos, la lucha contra la corrupción y la igualdad entre los ciudadanos (Bello, 2014).

Rastreamos en estos siete diarios los cuatro casos de suicidio durante once meses, tras trascender públicamente. Accedimos a las informaciones mediante el buscador de la web de cada medio. El criterio de búsqueda siempre fue el mismo: introdujimos el nombre de la persona en la barra de búsqueda para 
aseguramos hallar todas las informaciones sobre ella por su muerte, aunque no se mencionara de forma explícita el suicidio.

Los libros de estilo son una figura de autocontrol extendida. Suelen incluir pasajes que establecen el modo de actuar de sus redactores. Por ello, revisamos los seguidos por los siete diarios para identificar las referencias al suicidio (tabla 2).

Tabla 2. Normas para el tratamiento del suicidio de los libros de estilo de los medios analizados.

\begin{tabular}{|c|c|c|}
\hline Diario & Libro de estilo & Referencia al tratamiento del suicidio en su libro de estilo \\
\hline Clarín & $\begin{array}{l}\text { Manual de Estilo Clarín } \\
\text { (1997) }\end{array}$ & $\begin{array}{l}\text { «Las informaciones relativas a suicidios deberán publicarse solamente } \\
\text { cuando se trate de personas de relevancia o supongan un hecho social } \\
\text { de interés general. Este tipo de noticias deben tratarse con cuidado, ya } \\
\text { que pueden promover la imitación en personas en situación de riesgo } \\
\text { de quitarse la vida» (Manual de Estilo Clarín, 1997: 18). }\end{array}$ \\
\hline La Nación & $\begin{array}{l}\text { Manual de estilo y ética } \\
\text { periodística. La Nación } \\
\text { (1997) }\end{array}$ & $\begin{array}{l}\text { «No se han de ocultar las noticias sobre suicidios, pero se evitará la } \\
\text { difusión de detalles macabros, en la medida en que no sean necesarios } \\
\text { para que la información tenga sentido, esté bien calibrada y quede } \\
\text { completa» (Manual de Estilo Clarín, 1997: 30). }\end{array}$ \\
\hline$A B C$ & $\begin{array}{l}\text { Libro de Estilo de } A B C \\
\text { (Vigara, 2001) }\end{array}$ & - \\
\hline El Mundo & $\begin{array}{l}\text { Libro de Estilo de El } \\
\text { Mundo } \\
\text { (2002) }\end{array}$ & $\begin{array}{l}\text { «Un suicidio no debe ser noticia en sí mismo. Acaba siéndolo cuando } \\
\text { el autor es un personaje relevante o cuando se convierte en un hecho } \\
\text { significativo por la forma de llevarse a cabo, la edad o el problema } \\
\text { social que se esconda detrás. A la hora de informar de un suicidio hay } \\
\text { que tener en cuenta previamente dos cuestiones. Primera, que hay que } \\
\text { valorar el común criterio de psiquiatras y psicólogos que nos advierten } \\
\text { de que la noticias sobre un suicidio atrae a otras potenciales víctimas. } \\
\text { Segunda, que no hay que precipitarse y calificar de suicidio una muerte } \\
\text { solo por las apariencias. Hace falta profundizar en la noticia» (Libro de } \\
\text { Estilo de El Mundo, 2002: 67). }\end{array}$ \\
\hline El País & $\begin{array}{l}\text { Libro de Estilo El País } \\
\text { (2014) }\end{array}$ & $\begin{array}{l}\text { «El periodista deberá ser especialmente prudente con las } \\
\text { informaciones relativas a suicidios. En primer lugar, porque no siempre } \\
\text { la apariencia coincide con la realidad, y también porque la psicología } \\
\text { ha comprobado que estas noticias incitan a quitarse la vida a personas } \\
\text { que ya eran propensas al suicidio y que sienten en ese momento un } \\
\text { estímulo de imitación. Los suicidios deberán publicarse solamente } \\
\text { cuando se trate de personas de relevancia o supongan un hecho social } \\
\text { de interés general» (Libro de Estilo El País, 2014: 22). }\end{array}$ \\
\hline $\begin{array}{l}\text { El } \\
\text { Comercio }\end{array}$ & $\begin{array}{l}\text { Manual de estilo El } \\
\text { Comercio (Grupo El } \\
\text { Comercio, s.f.) }\end{array}$ & - \\
\hline $\begin{array}{l}\text { La } \\
\text { República }\end{array}$ & - & - \\
\hline
\end{tabular}

Fuente: elaboración propia.

Para el registro y posterior análisis de las informaciones diseñamos una ficha integrada por 15 variables: cinco descriptivas y 10 analíticas (tabla 3): 
Tabla 3. Ficha de análisis con las variables. Entre paréntesis aparecen las referencias documentales de las recomendaciones.

\begin{tabular}{|c|c|c|}
\hline \multicolumn{3}{|l|}{ Número de pieza. } \\
\hline Titular. & & \\
\hline \multicolumn{3}{|l|}{ Hipervínculo. } \\
\hline \multicolumn{3}{|l|}{ Medio. } \\
\hline \multicolumn{3}{|l|}{ Fecha. } \\
\hline & Sí & No \\
\hline \multicolumn{3}{|l|}{ 1. Alude al suicidio. } \\
\hline \multicolumn{3}{|l|}{ 2. Aparece explícitamente la palabra suicidio. } \\
\hline \multicolumn{3}{|l|}{$\begin{array}{l}\text { 3. Aporta información útil de cómo buscar ayuda (OMS, 2000, 2008, 2017; Ministerio de } \\
\text { Salud del Gobierno de Argentina, 2016; Ministerio de Sanidad, Política Social e Igualdad, } \\
\text { 2012). }\end{array}$} \\
\hline \multicolumn{3}{|l|}{$\begin{array}{l}\text { 4. Nombra motivaciones o problemas que expliquen el suicidio de forma simplista (OMS, } \\
\text { 2000, 2008, 2017; Ministerio de Salud del Gobierno de Argentina, 2016; Ministerio de } \\
\text { Sanidad, Política Social e Igualdad, 2012). }\end{array}$} \\
\hline \multicolumn{3}{|l|}{$\begin{array}{l}\text { 5. Explicita la forma de suicidio (OMS, 2000, 2008, 2017; Ministerio de Salud del } \\
\text { Gobierno de Argentina, 2016; Ministerio de Sanidad, Política Social e Igualdad, 2012). }\end{array}$} \\
\hline \multicolumn{3}{|l|}{ 6. Presupone suicidio antes de que se confirme por autopsia (OMS, 2008). } \\
\hline \multicolumn{3}{|l|}{ 7. Aparece la palabra suicidio en el titular (OMS, 2008, 2017). } \\
\hline \multicolumn{3}{|l|}{ 8. Profundiza y explica los problemas psiquiátricos subyacentes (OMS, 2000, 2008). } \\
\hline \multicolumn{3}{|l|}{ 9. Cuenta con fuentes expertas en psiquiatría (OMS, 2008, 2017). } \\
\hline 10. Engrandece o mitifica la figura del suicida (OMS, 2000, 2008, 2017). & & \\
\hline
\end{tabular}

Fuente: elaboración propia.

Establecimos las diez variables analíticas basándonos en las recomendaciones de los tres informes de la OMS (2000, 2008 y 2017), en la Guía de Práctica Clínica de Prevención y Tratamiento de la Conducta Suicida del Ministerio de Sanidad, Política Social e Igualdad (2012) y en las Recomendaciones para el Tratamiento de Temas de Salud Mental en los Medios de Comunicación del Ministerio de Salud del Gobierno de Argentina (2016).

No diferenciamos entre noticias procedentes de agencia, firmadas por el medio y firmadas por los redactores, puesto que su publicación revelaba la coherencia editorial y estilística con el diario.

También contactamos con los siete medios seleccionados para conseguir información complementaria, mediante entrevistas a integrantes de las redacciones, que permitieran conocer cómo tratan las informaciones sobre suicidios de personas famosas (tabla 4). Solo nos respondieron $A B C$ y El País. 
Tabla 4. Respuesta a las entrevistas solicitadas organizadas por diario.

\begin{tabular}{|l|l|l|l|}
\hline \multicolumn{1}{|c|}{ Diario } & \multicolumn{1}{|c|}{ Vía de contacto } & $\begin{array}{l}\text { Entrevista } \\
\text { solicitada }\end{array}$ & \multicolumn{1}{c|}{ Particularidades } \\
\hline Clarín & $\begin{array}{l}\text { Llamada telefónica, correo } \\
\text { electrónico y formulario web. }\end{array}$ & No concedida. & $\begin{array}{l}\text { No hubo respuesta al correo electrónico ni al } \\
\text { formulario web. Hablamos por teléfono con la } \\
\text { sección Espectáculos. Cuando expusimos el } \\
\text { tema de la investigación devolvieron la llamada } \\
\text { a la centralita. }\end{array}$ \\
\hline La Nación & $\begin{array}{l}\text { Llamada telefónica y correo } \\
\text { electrónico. }\end{array}$ & No concedida. & No hubo respuesta. \\
\hline ABC & $\begin{array}{l}\text { Llamada telefónica y correo } \\
\text { electrónico. }\end{array}$ & Concedida. & $\begin{array}{l}\text { Entrevistamos por teléfono a Isabel Gutiérrez } \\
\text { Rico, jefa de sección de Gente y Estilo de ABC. }\end{array}$ \\
\hline El Mundo & $\begin{array}{l}\text { Llamada telefónica y correo } \\
\text { electrónico. }\end{array}$ & No concedida. & $\begin{array}{l}\text { Romualdo Izquierdo, jefe de sección de La Otra } \\
\text { Crónica (LOC) de El Mundo, rechazó participar } \\
\text { en la investigación. }\end{array}$ \\
\hline El País & $\begin{array}{l}\text { Llamada telefónica y correo } \\
\text { electrónico. }\end{array}$ & Concedida. & $\begin{array}{l}\text { Entrevistamos por teléfono a Mábel Galaz, jefa } \\
\text { de la sección de Gente de El País. }\end{array}$ \\
\hline El Comercio & $\begin{array}{l}\text { Llamada telefónica y correo } \\
\text { electrónico. }\end{array}$ & No concedida. & No hubo respuesta. \\
\hline La República & $\begin{array}{l}\text { Llamada telefónica y correo } \\
\text { electrónico. }\end{array}$ & No concedida. & $\begin{array}{l}\text { Nuestro correo electrónico fue remitido, por } \\
\text { una secretaria, a la sección Espectáculos. No } \\
\text { respondieron. Contactamos por teléfono con la } \\
\text { misma sección. Reenviamos las preguntas por } \\
\text { correo electrónico. No respondieron. }\end{array}$ \\
\hline
\end{tabular}

Fuente: elaboración propia.

\section{Análisis de resultados}

El número de piezas periodísticas analizadas fue 582, lo que garantiza la validez estadística. Estudiamos los cuatro casos de suicidio en seis de los siete diarios. La excepción fue $E l$ Comercio, periódico peruano en el que solo registramos dos, el de Chester Bennington y el de Robin Williams. La causa es que su sistema de búsqueda hemerográfica no permite acceder a textos anteriores a 2014 (tabla 5).

Tabla 5. La distribución de las piezas por cabecera y países queda recogida en la N: número total de informaciones. $\bar{X}$ media.

\begin{tabular}{|c|c|c|c|c|c|c|c|c|c|c|}
\hline \multirow[t]{2}{*}{ Diarios } & \multirow[t]{2}{*}{$\begin{array}{c}\text { Inform. } \\
\text { analizadas }\end{array}$} & \multirow[t]{2}{*}{$\begin{array}{l}\text { Inform. } \\
\text { sobre } \\
\text { suicidio }\end{array}$} & \multirow[t]{2}{*}{$\begin{array}{l}\text { Contienen } \\
\text { la palabra } \\
\text { suicidio }\end{array}$} & \multirow[t]{2}{*}{ País } & \multicolumn{2}{|c|}{$\begin{array}{l}\text { Inform. } \\
\text { totales } \\
\text { analizadas }\end{array}$} & \multicolumn{2}{|c|}{$\begin{array}{c}\text { Inform. } \\
\text { totales } \\
\text { sobre } \\
\text { suicidio }\end{array}$} & \multicolumn{2}{|c|}{$\begin{array}{l}\text { Inform. } \\
\text { con la palabra } \\
\text { suicidio }\end{array}$} \\
\hline & & & & & $\mathbf{N}$ & $\overline{\mathrm{X}}$ & $\mathbf{N}$ & $\overline{\mathrm{X}}$ & $\mathbf{N}$ & $\overline{\mathrm{X}}$ \\
\hline Clarín & 34 & 26 & 22 & \multirow{2}{*}{ Argentina } & \multirow{2}{*}{140} & \multirow{2}{*}{70} & \multirow{2}{*}{74} & \multirow{2}{*}{37} & \multirow{2}{*}{50} & \multirow{2}{*}{25} \\
\hline La Nación & 106 & 48 & 28 & & & & & & & \\
\hline$A B C$ & 128 & 69 & 58 & \multirow{3}{*}{ España } & \multirow{3}{*}{247} & \multirow{3}{*}{82,33} & \multirow{3}{*}{149} & \multirow{3}{*}{49,7} & \multirow{3}{*}{123} & \multirow{3}{*}{41} \\
\hline El Mundo & 70 & 48 & 37 & & & & & & & \\
\hline El País & 49 & 32 & 28 & & & & & & & \\
\hline El Comercio & 123 & 68 & 63 & \multirow{2}{*}{ Perú } & \multirow{2}{*}{195} & \multirow{2}{*}{97,5} & \multirow{2}{*}{110} & \multirow{2}{*}{55} & \multirow{2}{*}{97} & \multirow{2}{*}{48,5} \\
\hline La República & 72 & 42 & 34 & & & & & & & \\
\hline
\end{tabular}

Fuente: elaboración propia. 
Observamos que el menor número de informaciones corresponde a los dos diarios de Argentina y el mayor a los dos de Perú. Son datos que muestran la tendencia de los medios de ambos países a tratar el suicidio sin recurrir a eufemismos. La existencia de un código deontológico restrictivo como es el del Foro de Periodismo Argentino (FOPEA), junto con los libros de estilo de los dos diarios argentinos, propicia que los suicidios no se traten tanto como en los diarios de España y Perú. Los medios argentinos no solo evitan informar de estas muertes, sino que esquivan la palabra suicidio utilizando rodeos como «poner fin a su sufrimiento», «decidir pasar a mejor vida», «terminar con todo» 0 «acabar con su vida».

Independientemente del país de cada diario, y de si disponen o no de autorregulación específica, detectamos cuatro tendencias generales:

$\rightarrow 1$. No se ofrece información de cómo buscar ayuda si se encuentran en riesgo de suicidio. Solo la identificamos en una de las 582 informaciones.

$\rightarrow 2$. Solo en siete ocasiones de las 582 analizadas, los diarios cuentan con fuentes expertas en psiquiatría que contextualizan la influencia de las enfermedades mentales en las personas y que «eduquen a la población» (Ministerio de Sanidad, Política Social e Igualdad, 2012: 160).

$\rightarrow 3$. Solo en ocho de los 582 casos, los periodistas realizan labor pedagógica. En todos los casos estudiados, la variable «profundiza y explica los problemas psiquiátricos subyacentes» está representada de forma minoritaria.

$\rightarrow 4$. La mayoría de los titulares no contienen la palabra suicidio. Esta aparece en 40 de las 582 informaciones. Es un dato que concuerda con las recomendaciones de los profesionales de la salud. Estos titulares se distribuyen de forma similar entre las siete cabeceras estudiadas. Es una diferencia destacable si tenemos en cuenta otros estudios publicados (Young et al., 2017).

Respecto a la adecuación a las recomendaciones de los expertos en salud y sanidad, si analizamos las cifras medias (tabla 6) podemos afirmar que existe una diferencia considerable entre los medios peruanos y los de los otros dos países. Los medios peruanos son los que más se acercan a ellas. Solo en la variable «Menciona motivación o problema que explique el suicidio de forma simplista» los medios peruanos superan a los argenti- 
nos en un 12,49 por ciento, pues el porcentaje de estos últimos es singularmente bajo en esta variable.

Tabla 6. Comparativa porcentual y medias de cuatro variables fuertes de las cabeceras por países.

\begin{tabular}{|l|r|r|r|r|r|r|r|}
\hline & Clarín & La Nación & ABC & El Mundo & El País & $\begin{array}{c}\text { El } \\
\text { Comercio }\end{array}$ & $\begin{array}{c}\text { La } \\
\text { República }\end{array}$ \\
\hline $\begin{array}{l}\text { Menciona motivación 0 } \\
\text { problema que explique } \\
\text { el suicidio de forma } \\
\text { simplista. }\end{array}$ & $39,98 \%$ & $15,08 \%$ & $51,33 \%$ & $55,88 \%$ & $63,68 \%$ & $29,42 \%$ & $50,61 \%$ \\
\hline $\begin{array}{l}\text { Explicita la forma de } \\
\text { suicidio. }\end{array}$ & $62,83 \%$ & $42,71 \%$ & $61,19 \%$ & $54,46 \%$ & $73,25 \%$ & $30,89 \%$ & $51,98 \%$ \\
\hline $\begin{array}{l}\text { Presupone suicidio } \\
\text { antes de que se } \\
\text { confirme. }\end{array}$ & $85,58 \%$ & $81,88 \%$ & $79,10 \%$ & $76,55 \%$ & $65,94 \%$ & $77,96 \%$ & $67,79 \%$ \\
\hline $\begin{array}{l}\text { Engrandece o mitifica } \\
\text { la figura del suicida. }\end{array}$ & $28,33 \%$ & $57,00 \%$ & $47,63 \%$ & $48,51 \%$ & $42,10 \%$ & $48,55 \%$ & $26,40 \%$ \\
\hline
\end{tabular}

\begin{tabular}{|l|r|r|r|}
\hline & \multicolumn{1}{|c|}{ Diarios argentinos } & Diarios españoles & \multicolumn{1}{|c|}{ Diarios peruanos } \\
\hline $\begin{array}{l}\text { Menciona motivación } \\
\text { problema que explique } \\
\text { el suicidio de forma } \\
\text { simplista. }\end{array}$ & $27,53 \%$ & $56,96 \%$ & $40,02 \%$ \\
\hline $\begin{array}{l}\text { Explicita la forma de } \\
\text { suicidio. }\end{array}$ & $52,77 \%$ & $62,97 \%$ & $41,44 \%$ \\
\hline $\begin{array}{l}\text { Presupone suicidio } \\
\text { antes de que se } \\
\text { confirme. }\end{array}$ & $83,73 \%$ & $73,86 \%$ & $72,88 \%$ \\
\hline $\begin{array}{l}\text { Engrandece o mitifica } \\
\text { la figura del suicida. }\end{array}$ & $42,665 \%$ & $46,08 \%$ & $37,48 \%$ \\
\hline
\end{tabular}

Fuente: elaboración propia.

Las cifras de los diarios peruanos muestran que son los que más se ciñen a las recomendaciones de los expertos (tabla 6). Este resultado coincide con que el Código de Ética de la Federación de Periodistas del Perú (Vignolo, 2001) aconseje cautela, evitar el escándalo y el sensacionalismo, aunque sin limitar las informaciones sobre suicidios. Además, el diario El Comercio, el único de los dos medios peruanos con libro de estilo, no restringe la cobertura de estas muertes.

Las entrevistas realizadas a Isabel Gutiérrez Rico, de $A B C$, y a Mábel Galaz, de El País, permiten conocer cómo se procede en ambos diarios al informar del suicidio de un personaje famoso. El País aplica solo su libro de estilo y $A B C$, cuyo libro de estilo no alude al suicidio, se atiene a los límites legales. No siguen las recomendaciones de los profesionales de la salud. Se observa el efecto del Libro de Estilo El País (2014), frente al de $A B C$ (Vigara, 2001). 
De los tres medios españoles, El País es el diario con menos informaciones sobre suicidio, 32. En $A B C$ ascienden a 69, la cifra mayor de los siete diarios. Le sigue $\mathrm{El}$ Comercio con 68. Esto podría explicarse porque, según Isabel Gutiérrez Rico, de $A B C$, en los casos de famosos internacionales actúan con mayor libertad, haciéndose eco de las informaciones emitidas por otros medios y de las recibidas de las agencias de noticias.

Ni El País ni $A B C$ se remiten a las recomendaciones de los profesionales de la salud. De hecho, de los siete diarios, El País es el que más veces especifica el método de suicidio utilizado, un 73,25 por ciento de los casos. Es el diario español que más veces menciona motivaciones o problemas que explican el suicidio de forma simplista.

Si comparamos las medias porcentuales de España y Argentina (tabla 6), los diarios digitales argentinos realizan un tratamiento de las noticias de suicidio más acorde con las recomendaciones de los expertos; pero la diferencia es tan reducida que no es significativa. La diferencia se amplía en la variable «Menciona motivación o problema que explique el suicidio de forma simplista». En esta variable, los diarios españoles superan a los argentinos en casi un 30 por ciento.

En los ítems restantes, los datos de los medios españoles y argentinos son similares (tabla 6). Los argentinos superan a los españoles en un 9,87 por ciento en la variable referida a presuponer el suicidio como causa de la muerte antes de que lo confirme la autopsia.

Siguiendo con las medias porcentuales que revelan la tendencia general, es cierto que en los tres países hay medios que, en ocasiones, no se ajustan en absoluto a las recomendaciones de los expertos en salud. De hecho, los siete diarios deberán aplicar ajustes de calado para que sus informaciones sigan esas recomendaciones de la OMS.

\section{Discusión y conclusiones}

Para abordar este estudio revisamos las normas de autorregulación periodísticas referidas al suicidio (FOPEA, FPP, FAPE), investigaciones hemerográficas sobre suicidios de personajes famosos realizadas por psiquiatras (v.g. Chen et al., 2007; Ju Ji et al., 2014; Park et al., 2016) y las recomendaciones de la OMS (2000, 2008, 2017). Esta consulta documental revela la preocupación de los especialistas en salud mental por el tratamiento mediático inadecuado del suicidio.

De hecho, los datos obtenidos en nuestro estudio confirman que existe una disonancia manifiesta entre dicho tratamiento y lo recomendado en los documentos de autorregulación y las guías internacionales de buenas prácticas. Estamos ante un problema de salud pública cuya magnitud requiere un cambio 
de actitud periodística. Ese cambio de actitud de los medios subsanaría la falta de criterio y la confusión que persisten al informar sobre suicidios. Y, probablemente, el periodo de formación de los estudiantes de periodismo sea un ámbito propicio para impulsarlo.

Presenta interés comparar los resultados de este estudio con medios de comunicación de otras tradiciones periodísticas. El meta-análisis de casos de suicidio de personajes famosos ocurridos en ámbitos culturales diferentes ordenaría y sintetizaría lo que la literatura especializada recoge sobre países asiáticos, europeos y norteamericanos.

Nuestros resultados permiten extraer las conclusiones siguientes:

Los medios de comunicación realizan un tratamiento informativo inadecuado de los casos de suicidio de personas famosas, sin ceñirse a las recomendaciones elaboradas por las instituciones sanitarias y los expertos en psiquiatría, asequibles a periodistas y medios. La H1 queda confirmada y se cumple el O1.

Los países con códigos éticos o deontológicos que aluden al suicidio (Argentina-FOPEA y Perú-FPP) no muestran diferencias significativas con el país que carece de ese referente (España-FAPE). Esto se debe a que las normas de los códigos deontológicos argentino y peruano no se ajustan a las recomendaciones de los expertos en salud. Sus enunciados genéricos y superficiales soslayan esas recomendaciones y no contribuyen a mejorar el tratamiento del suicidio. La $\mathrm{H} 2$ queda verificada y el $\mathrm{O} 2$ cumplido.

No se observa una diferencia significativa entre los medios de comunicación con libro de estilo que pauta el tratamiento del suicidio (Clarín, La Nación, El Mundo, El País) y los que no disponen de él (ABC, El Comercio, La República), al contrario de lo que cabía esperar. Las variables que muestran diferencias vienen asociadas a un código ético o deontológico nacional que incluye normas que, a su vez, podrían estar reforzando las del libro de estilo propio. La $\mathrm{H} 3$ queda refutada y el $\mathrm{O} 3$ cumplido.

Los diarios analizados muestran que ni periodistas ni responsables de medios han modificado las prioridades ni los enfoques obsoletos al tratar el suicidio. Priorizan no ocasionarse contratiempos legales. Prima la cobertura sensacionalista en lugar de comprometerse con enfoques pedagógicos y preventivos exigibles al periodismo actual.

La especialización periodística en salud y sanidad ofrece, junto con la formación en deontología, la capacitación profesional destinada a transformar tratamientos informativos hoy inadecuados en otros que orienten al público y ayuden a personas en situación de riesgo.

Las enfermedades mentales comprometen a miles de vidas cada año en numerosos países y convierten el suicidio en una de las primeras causas de 
muerte. La reflexión sobre cómo tratar este asunto está presente, aunque de forma genérica, incluso en las asociaciones de periodística de países desarrollados con tasas de suicidio que duplican a la española $\left(\mathrm{vg}\right.$. Noruega ${ }^{2}, \mathrm{Suecia}^{3}$, Finlandia ${ }^{4}$ ). Esta situación exige un periodismo que supere el impacto del drama en favor de la calidad informativa, haciendo suyas las recomendaciones de la OMS.

La situación revelada aquí nos lleva a sugerir la inclusión en los estudios de Periodismo de unos contenidos formativos mínimos sobre el tratamiento del suicidio. Materias que suelen cursar los alumnos, como Comunicación de la ciencia, Periodismo Científico, Periodismo especializado, Ética o Deontología periodística constituyen marcos curriculares adecuados para ello.

\section{Referencias bibliográficas}

Acosta, F. J.; Rodríguez C. J. y Cejas, M. R. (2017). Noticias sobre suicidio en los medios de comunicación. Recomendaciones de la OMS. Rev Esp Salud Pública, 91(octubre), e1-e7. Recuperado de: RS91C_201710040.pdf

Accountable Journalism (s.f.a). Asociación de Entidades Periodísticas Argentinas: Code of Ethics of the Argentine Press. Recuperado de: https:/laccountablejournalism.org/ethics-codes/asociacion-de-entidades-periodisticas-argentinas-code-of-ethics-of-the-arge

Accountable Journalism (s.f.b). Code of Ethics of the Association of Journalists of Guatemala. Recuperado de: https:/laccountablejournalism.org/ethics-codes/GuatemalaDeclaration

Accountable Journalism (s.f.c). Mexico: Journalists' Code of Ethics. Recuperado de: https:/laccountablejournalism.org/ethics-codes/Mexico-Journalist

Accountable Journalism (s.f.d). Code of Ethics of the National Association of Journalists of Peru. Recuperado de: https://accountablejournalism.org/ethics-codes/Peru-ANP

APES (2012). Código de ética para los periodistas y profesionales de la comunicación en El Salvador. Asociación de Periodistas de El Salvador. San Salvador: APES. Recuperado de: http://apes.org.sv/wordpress/wp-contentluploads/2014/10/ CODIGO-DE-ÉTICA.pdf

BArbero, M. (1966). El Suicidio. Madrid: Taurus Ediciones.

Beam, R. A.; John, S. L. y Yaqub, M. M. (2018). We Don't Cover Suicide... (Except When We Do Cover Suicide). A case study in the production of news. Journalism Studies, 19(10), 1.447-1.465. Doi:10.1080/146167 0X.2017.1279563

[02] Punto 4.9 de: https://presse.no/pfu/etiske-regler/vaer-varsom-plakaten/vvpl-engelsk/

[03] Punto 8 en: https://www.sjf.se/yrkesfragor/yrkesetik/spelregler-press-radio-och-tv/publicitetsregler

[04] Punto 28 referido a enfermedades y muertes en: https://journalistiliitto.fi/fi/pelisaannot/journalistin-ohjeet/ 
Bello, N. (2014). Y el Perú, iay! iseguía choleando!: análisis de dos casos de discriminación social en cuatro diarios limeños (tesis doctoral). Recuperado de: http://tesis.pucp.edu.pe/repositorio/handle/123456789/5656

Bonete, E. (1995). De la ética filosófica a la deontología periodística, en E. Bonete Perales (ed.), Éticas de la información y deontologías del Periodismo (pp. 17-49). Madrid: Tecnos.

CAmps, V. (1995). El lugar de la ética en los medios de comunicación, en E. Bonete Perales (ed.), Éticas de la información y deontologías del Periodismo (pp. 53-64). Madrid: Tecnos.

Cheng, A. T. A.; Hawton, K.; Lee, C. T. C. y Chen, T. H. H. (2007). The influence of media reporting of the suicide of a celebrity on suicide rates: a population-based study. International Journal of Epidemiology, 36(6), 1.229-1.234. Doi:10.1093/ije/dym196

Clemente, M. y GonzÁlez, A. (1996). Suicidio, una alternativa social. Madrid: Biblioteca Nueva.

Código de Ética (1994). Colegio Dominicano de Periodistas. Santo Domingo: CDP. Recuperado de: http://www.unesco.org/new/fileadmin/MULTIMEDIA/HQ/C//Climages/ wmn/code $\% 20$ of $\% 20$ Ethics $\% 20$ Collegio $\% 20$ de $\% 20$ periodistas.pdf

Código de ética de FOPEA (2006). Buenos Aires: Foro de Periodismo Argentino. Recuperado de: http://www.fopea.org/etica-y-calidad/codigo-de-etica-de-fopeal

Código de ética de la Asociación Nacional de Periodismo de Bolivia (2007). Tarija: ANP. Recuperado de: http://www.anp-bolivia.com/tae-anp-tribunal-de-autorregulacionetica-de-la-asociacion-nacional-de-la-prensa-de-bolivial

Código de ética de las y los profesionales de la comunicación (2011). Colegio de Periodistas de Costa Rica. Recuperado de: http://www.colper.or.cr/userfiles/file/juridicol codigos/codigo_etica.pdf

Código de Ética Periodística (2007). Panamá: Fórum de Periodistas por las libertades de expresión e Información. Recuperado de: http://www.forumdeperiodistas.org/2495-2/

Código de Ética Periodística (2013). Asociación de la Prensa Uruguaya. Recuperado de: http://etica.cainfo.org.uy/codigo-de-etica-periodistica/

Código de Ética de Periodistas de Chile (2015). Colegio de Periodistas de Chile. Arica: CPC. Recuperado de: https://www.colegiodeperiodistas.cl/pletica-periodistica.html

Código de Ética del Círculo de Periodistas de Bogotá (2006). Círculo de Periodistas de Bogotá. Recuperado de: https://www.circuloperiodistasdebogota.org/comision-de-etical

Código de Ética del Periodista (2013). Unión de Periodistas de Cuba. Recuperado de: http://www.cubaperiodistas.culindex.php/codigo-de-etica-del-periodistal

Código de ética del Periodista y Comunicador de Guatemala (2015-2016). Cámara Guatemalteca de Periodismo. Recuperado de: http://cgp.org.gt/codigo-de-etical 
Código de Ética del Periodista Venezolano (2013). Colegio Nacional de Periodistas. Mérida: CNP. Recuperado de: http://cdn1.cnpven.org/archivos/80/original_codigoeticafinal.pdf

Código de Ética del Sindicato de Periodistas del Paraguay (1999). Asunción: Sindicato de Periodistas del Paraguay (SPP). Recuperado de: http://www.sindicatodeperiodistas.org.py/wp-content/uploads/2018/07/Código-de-Ética-del-Sindicato-de-Periodistas-del-Paraguay.pdf Colegio de Periodistas de Honduras (1979). Código de Ética. Recuperado de: http://colegiodeperiodistasdehonduras.hn/wp/2017/11/23/codigo-de-etica-del-colegio-de-periodistasde-honduras/

Comisión de las Comunidades Europeas (2005). Libro Verde. Recuperado de: https:/lec.europa.eu/health/ph_determinants/life_style/mental/green_paper/mental_gp_es.pdf

ComScore (2017). comScore Anuncia Mejoras Significativas en MMX Multiplataforma y Mobile Metrix en Argentina con la introducción del Panel de Datos del Consumidor Móvil. Recuperado de: https://www.comscore.com/lat/Prensa-yEventos/Comunicados-de-prensa/2017/6/comScore-Anuncia-Mejoras-Significativas-en-MMX-Multiplataforma-y-Mobile-Metrix-en-Argentina-con-la-introduccion-del-Panel-de-Datos-del-Consumidor-Movil

De la Vega, G. (1996). Crítica del suicidio con un fundamento ético aristotélico. Breve reflexión filosófica. Anales Médicos, 41(3), 129-130.

Díaz del Campo-Lozano, J. y Chaparro-Domínguez, M. A. (2018). Los desafíos éticos del periodismo en la era del big data: análisis de códigos deontológicos latinoamericanos. Palabra Clave, 21(4), 1136-1163. Doi: 10.5294/pacla.2018.21.4.8

FAPE (2017). Código Deontológico. Federación de Asociaciones de Periodistas de España. Mérida: FAPE. Recuperado de: http://fape.es/home/codigo-deontologicol

Federación Nacional de Periodistas del Ecuador (1980). Código de Ética del Periodista. Cuenca: FENAPE. Recuperado de: https:/leticadelacomunicacion.wordpress.com/2008/06/18/codigo-de-etica-periodistica-en-el-ecuador/

García, M. (2011). La fantasía neutral: el diario El Comercio y las estrategias discursivas del poder en el Perú (tesis doctoral). Recuperado de: http:/ltesis.pucp. edu.pe/repositorio/handle/123456789/1395

Garrido-Fabián, F.; Serrano-López, A.E. y Catalán-Matamoros, D. (2018). El uso por los periodistas de las recomendaciones de la OMS para la prevención del suicidio. El caso del periódico ABC. Revista Latina de Comunicación Social, 73, 810-827.

Herrera, R.; Ures, M.B. y Martínez, J. J. (2014). El tratamiento del suicidio en la prensa española: ¿efecto werther o efecto papageno? Rev. Asoc. Esp. Neuropsiq., 35(125), 123-134.

Herrero, M.; Artero, J.P. y SÁnchez-TABerno, A. (2009). Los contenidos editoriales como herramienta determinante para construir una marca: el caso de $E l$ Mundo. Doxa Comunicación, 8, 163-183. 
IAB Perú (2018). ComScore presenta «Perú Media Index 2018». Recuperado de: https://iabperu.com/news/comscore-presenta-peru-media-index-2018

Jorm, A. F.; Barney, L. J.; Christensen, H.; Highet, N. J.; Kelly, C. M. y KitchENER, B. (2006). Research on mental health literacy: what we know and what we still need to know. Australian and New Zealand Journal of Psychiatry, 40(1), 3-5.

Ju Ji, N.; Young Lee, W.; SeoK Noh, M.; y YiP, P. S. F. (2014). The impact of indiscriminate media coverage of a celebrity suicide on a society with a high suicide rate: Epidemiological findings on copycat suicides from South Korea. Journal of Affective Disorders, 156, 56-61. Doi:10.1016/j.jad.2013.11.015

Libro de Estilo de El Mundo (2002). Madrid: El Mundo. Recuperado de: http://www.masmenos.es/wp-content/uploads/2002/01/librodeestilo_elmundo.pdf

Libro de Estilo El País (1996). Madrid: Ediciones El País.

Libro de Estilo El País (2014). Madrid: Ediciones El País.

Lois Barcia, M.; Rodríguez Arias, I.; TúÑez López, J. M. (2018). Pautas de redacción y análisis de contenido en noticias sobre suicidio en la prensa española e internacional: efecto Werther\&Papageno y seguimiento de las recomendaciones de la OMS. Zer: Revista de estudios de comunicación, 23(45); 139-159. Doi:10.1387/zer.20244

LóPEz, M. M. (2002). La información sobre el dolor humano. Del morbo al respeto, en J. A. Agejas y F. J. SerRano (coords.), Ética de la Comunicación y de la información (pp. 177-192). Barcelona: Ariel.

LóPez, M. M. (2016). Ética en los medios de comunicación. Prensa, radio, TVy cine. Barcelona: Editorial UOC.

LóPEZ MAÑERo, C. (1997). Criterios deontológicos en el tratamiento informativo del dolor. Comunicación y sociedad, 10(2), 125-161.

Manual de Estilo Clarín (1997). Buenos Aires: Grupo Clarín. Recuperado de: https://grupoclarin.com/sites/defaultffiles/manual_de_estilo.pdf

Manual de estilo El Comercio (s.f.). Quito: Grupo El Comercio. Recuperado de: https://www.grupoelcomercio.com/images/stories/Descargables/manual_de_estilo.pdf

Manual de estilo y ética periodística. La Nación (1997). Buenos Aires: Espasa Calpe. Recuperado de: http://www.mdp.edu.ar/humanidades/documentacion/licad/archivos/ modulos/inicial/archivos/bibliografia/inicial/MI019.pdf

Ministerio de Sanidad, Política Social e Igualdad (2011). Estrategia en Salud Mental del Sistema Nacional de Salud 2009-2013. Madrid. Recuperado de: https://www.msssi.gob.es/organizacion/sns/planCalidadSNS/pdf/excelencia/salud_mental/ESTRATEGIA_SALUD_MENTAL_SNS_PAG_WEB.pdf

Ministerio de Sanidad, Política Social e Igualdad (2012). Guía de Práctica Clínica de Prevención y Tratamiento de la Conducta Suicida. Recuperado de: http://www.guiasalud.es/GPC/GPC_481_Conducta_Suicida_Avaliat_compl.pdf 
Ministerio de Salud (2016). Recomendaciones para el Tratamiento de Temas de Salud Mental en los Medios de Comunicación. Buenos Aires. Recuperado de: http://www.msal.gob.arlimages/stories/bes/graficos/0000000479cnt-recomendaciones-tratamientotemas-salud-mental-medios.pdf

MüLleR, F. (2011). El 'efecto Werther'. Gestión de la información del suicidio por la prensa española en el caso de Antonio Flores y su repercusión en los receptores. Cuadernos de gestión de información. Revista académica interdisciplinar sobre gestión de información en las organizaciones, 1, 65-71.

MuÑoz, L. F. y SÁNCHEZ, R. (2014). Caracterización de noticias sobre suicidio en medios impresos en Colombia. Revista colombiana de Psiquiatría, 43 (S1), 12-18.

NiederkRotentHALER, T. et al. (2012). Changes in suicide rates following media reports on celebrity suicide: a meta-analysis. J Epidemiol Community Health, 66, 1.037-1.042.

NúÑEz, E. (1996). Código Deontológico Europeo de la Profesión Periodística. Recuperado de: http://www.asociacionprensa.org/es/images/Codigo_Deontologico_Europeo_de_ la_Profesion_Periodistica.pdf

Olmo, A. y García, D. (2014). El tratamiento de las noticias sobre suicidios. Una aproximación a su reflejo en los medios de comunicación. Estudios sobre el mensaje periodístico, 20(2), 1.149-1.163.

Olmo, A. (2015). Suicidio y libertad de información: Entre la relevancia pública y la responsabilidad. Revista de Comunicación de la SEECI, 19(38), 70-114. Doi: 10.15198/seeci.2015.38.70-114

Organización Mundial de la Salud (2000). Prevención del suicidio. Un instrumento para profesionales de los medios de comunicación. Ginebra: World Health Organization. Recuperado de: http://www.who.int/mental_health/media/media spanish.pdf

Organización mundial de la Salud (2008). Preventing Suicide. A Resource for Media Professionals. Ginebra: Word Health Organization.

Organización Mundial de la Salud (2014). Preventing suicide. A global imperative. Luxemburgo: World Health Organization. Recuperado de: http:/lapps.who. intliris/bitstream/handle/10665/131056/9789241564779_eng.pdf;jsessionid=CBEDD98DD411E9FA3553 A1252938DD00?sequence=1

Organización Mundial de la Salud (2017). World Health Statistics 2017: Monitoring health for the SDGs. Ginebra: World Health Organization. Recuperado de: http://www.who.int/gho/publications/world_health_statistics/2017/en/

Organización Mundial de la Salud (2018). World Health Statistics 2018: Monitoring health for the SDGs. Ginebra: World Health Organization. Recuperado de: http://apps.who.intliris/bitstream/handle/10665/272596/9789241565585-eng.pdf?ua=1 
Park, J.; Choi, N.; Kim, S. J.; Kim, S.; An, H.; Lee, H. J. y Lee, Y. J. (2016). The Impact of Celebrity Suicide on Subsequent Suicide Rates in the General Population of Korea from 1990 to 2010. Journal of Korean Medical Science, 31(4), 598. Doi:10.3346/jkms.2016.31.4.598

Phillips, D. (1974). The Influence of Suggestion on Suicide: Substantive and Theoretical Implications of the Werther Effect. American Sociological Review, 39(3), 340-354. Doi: 10.2307/2094294.

PIRKIS, J. y BLOOD, R. (2001). Suicide and the media. Part I: Reportage in nonfictional media. Crisis, 22, 146-154.

Rodríguez, L. (1995). Ética clásica y ética periodística, en E. Bonete Perales (ed.), Éticas de la información y deontologías del Periodismo (pp. 65-80). Madrid: Tecnos.

Rubiano, H.; Quintero, T. y Bonilla, I. (2007). El suicidio: más que una noticia, un tema de responsabilidad social. Palabra Clave, 10 (2), 93-111.

Statista (2017). Número de lectores diarios de los principales periódicos españoles en 2017 (en miles de lectores). Recuperado de: https:/les.statista.com/estadisticas/476795/periodicos-diarios-mas-leidos-en-espanal

UrzúA, M. (2003). Suicidio, intimidad e información. Documentación de las Ciencias de la Información, 26, 351-358.

Vigara Tauste, A. M. (2001). Libro de Estilo de ABC. Barcelona: Editorial Ariel. Vignolo, A. (2001). Código de Ética de la Federación de Periodistas del Perú. Lima: FPP. Recuperado de: http://www.periodistasdelperu.org/2009/10/codigo-de-etica-de-lafpp.html

WASSERMAN, I. (1984). Imitation and Suicide: A Reexamination of the Werther Effect. American Sociological Review, 49(3), 427-436. Doi: 10.2307/2095285

Young, R.; Subramanian, R.; Miles, S.; Hinnant, A. y Andsager, J. L. (2017). Social Representation of Cyberbullying and Adolescent Suicide: A MixedMethod Analysis of News Stories. Health Communication, 32(9), 1.0821.092. Doi: 10.1080/10410236.2016.1214214

Zunino, E. (2015). El conflicto político en los medios gráficos de la Argentina: un estudio de caso. Revista Mexicana de Opinión Pública, 12, 81-105.

Para citar este artículo: Granja-González, S. y Meneses-Fernández, M. D. (2019). El tratamiento periodístico del suicidio en diarios digitales de Argentina, España y Perú. index.comunicación, 9(1), 57-79. 
